\title{
Role of endothelin B receptor in oligodendroglioma proliferation and survival: In vitro and in vivo evidence
}

\author{
XIN WAN, LONGBO ZHANG and BING JIANG \\ Department of Neurosurgery, Xiangya Hospital, Central South University, Changsha, Hunan 410078, P.R. China
}

Received June 9, 2013; Accepted October 7, 2013

DOI: $10.3892 / \mathrm{mmr} .2013 .1746$

\begin{abstract}
In this study, the role of the endothelin B receptor (ETBR) in oligodendroglioma cell proliferation and survival was investigated in vitro and in vivo. The overexpression and knockdown of ETBR was conducted in Hs683 human oligodendroglioma cells, and cell proliferation and activation (phosphorylation) of extracellular signal-regulated kinase (ERK) were measured in vitro. An orthotopic xenograft oligodendroglioma mouse model was established. Mouse survival times and immunohistochemical Ki67 staining in the xenografts were examined. In vitro experiments demonstrated that the overexpression of ETBR significantly enhanced the proliferation of oligodendroglioma cells and the activation of ERK compared with the controls, which was eliminated by the selective ETBR inhibitor BQ788 and ERK-specific inhibitor U0126, but not selective endothelin A receptor inhibitor BQ123. By contrast, the knockdown of endogenous ETBR markedly decreased oligodendroglioma cell proliferation and the activation of ERK compared with the controls. Overexpression of ETBR significantly increased immunohistochemical Ki67 staining in the Hs683 cell orthotopic xenograft and decreased animal survival. By contrast, knockdown of ETBR significantly decreased Ki67 staining and increased mouse survival times. Intratumoral injection of BQ788, but not BQ123, significantly decreased Ki67 staining and prolonged mouse survival times. In conclusion, ETBR was demonstrated to mediate the proliferation of oligodendroglioma cells according to an ERK-dependent mechanism. Using an orthotopic xenograft oligodendroglioma mouse model, it was demonstrated in vivo that ETBR promotes oligodendroglioma proliferation and that the selective ETBR antagonist effectively inhibits the proliferation of oligodendroglioma cells and prolongs survival times. This study provides a novel insight into the role of ETBR in
\end{abstract}

Correspondence to: Professor Bing Jiang, Department of Neurosurgery, Xiangya Hospital, Central South University, 87 Xiangya Road, Changsha, Hunan 410078, P.R. China

E-mail:wanxin70108@sina.com

Key words: endothelin B receptor, endothelin, oligodendroglioma, proliferation, survival, Ki67, extracellular signal-regulated kinase, orthotopic xenograft, intratumoral injection oligodendroglioma proliferation and survival, and provides the first in vivo evidence that ETBR-specific antagonists are a potential therapeutic alternative for oligodendrogliomas.

\section{Introduction}

Oligodendrogliomas, which are predominantly composed of cells that morphologically resemble oligodendrocytes, are the third most common type of glioma, comprising 2-5\% of primary brain tumors and $4-15 \%$ of all gliomas $(1,2)$. The actual frequency of oligodendrogliomas may have been largely underestimated due to a of lack of specific markers for tumoral oligodendrocytes (1). As these tumors are capable of affecting regions of the brain that control speech, vision and motor functions, surgery may be associated with the risk of disability. Thus, an understanding of their molecular pathogenesis may provide important alternative therapeutic options (2). Furthermore, using current treatment strategies, oligodendrogliomas are considered to be incurable (3). The standard chemotherapy regimen for patients with oligodendroglioma involves a combination treatment with procarbazine, lomustine and vincristine. Each of these drugs was developed several decades ago $(4,5)$, and there is an urgent requirement for novel drugs or therapies (6).

The endothelin (ET) family includes three 21-amino acid peptides, ET-1, ET-2 and ET-3, that are released from their large precursor peptides, preproETs, by the action of ET-converting enzymes. ETs induce their effects via the stimulation of two G protein-coupled receptors: ET A receptor (ETAR) and ET B receptor (ETBR) (7). ETAR preferentially binds to ET-1, while ETBR binds to all three ETs with a similar affinity (1). ETAR mRNA appears to have a restricted distribution and is predominantly expressed in the vascular smooth muscle cells of peripheral tissues, bronchial smooth muscle cells, myocardium and the pituitary gland. By contrast, ETBR mRNA is more widely distributed, with prominent expression in the brain, pedominantly in glial cells (1). Previous studies have demonstrated the expression of the ET system in various types of gliomas (8). Egidy et al (9) reported that while ETAR was highly expressed in glioblastoma vessels and in certain scattered glioblastoma areas, ETBR was predominantly identified in tumor cells. Paolillo et al (10) revealed that human glioblastoma cell lines were capable of releasing ET-1 and expressing functional ETBR, while ETAR appeared to be absent or barely detectable. Paolillo et al (11) demonstrated that selective ETBR, but not ETAR, antagonists blocked proliferation 
in astrocytoma cells. Anguelova et al (1) reported that ETBR was predominantly detected in human oligodendrogliomas rather than in glioblastomas. The findings suggest that ETBR is important in glioma pathogenesis, particularly, oligodendrogliomas. In this study, the role of ETBR in oligodendroglioma proliferation and survival was investigated in vitro and in vivo.

\section{Materials and methods}

Cells line, reagents and mice. The Hs683 human glioma cell line (HTB-138) was purchased from the American Type Culture Collection (ATCC, Manassas, VA, USA). Human ETBR cDNA was subcloned into a pcDNA3.1 expression vector. ETBR (sc-270098-V) short hairpin RNA (shRNA) lentiviral particles, control shRNA lentiviral particles-A (sc-108080) and the anti-ETBR (N-21) (sc-21199) antibody were purchased from Santa Cruz Biotechnology (Santa Cruz, CA, USA). The anti-Ki67 antibody (ab15580) was purchased from Abcam (Hong Kong, China). BQ123, BQ788, G418, puromycin, extracellularsignal-regulated kinases-1 and2(ERK1/2)inhibitor U0126 and anti-ERK (M3807) and anti-phosphorylated ERK antibodies were purchased from Sigma (St. Louis, MO, USA). The Superfect ${ }^{\mathrm{TM}}$ transfection reagent was purchased from Qiagen (Valencia, CA, USA). The methlythiazoletetrazolium (MTT) cell proliferation and viability assay kit was purchased from R\&D Systems (Minneapolis, MN, USA). Eight-week-old $\mathrm{BALB} / \mathrm{C}$ female nude mice were purchased from Central South University (Changsha, China) and were housed at the Xiangya Hospital BioResources Centre (Changsha, China).

Transfection and lentiviral transduction. The ETBR expression construct was transfected into Hs683 cells using the Superfect transfection reagent according to the manufacturer's instructions. Pools of stable transfectants were generated via selection with G418 $(1.25 \mathrm{mg} / \mathrm{ml})$. ETBR shRNA lentiviral particles contain expression constructs, which encode a target-specific 19-25 nt (plus hairpin) shRNA, designed to specifically knockdown ETBR gene expression. Control shRNA lentiviral particles contained a scrambled shRNA sequence that did not lead to the degradation of any cellular mRNA, and was used as a negative control for ETBR shRNA lentiviral particles. Lentiviral transduction was performed in Hs683 cells. Pools of stable transductants were generated via selection with puromycin $(5 \mu \mathrm{g} / \mathrm{ml})$ according to the manufacturer's instructions (Santa Cruz Biotechnology).

In vitro cell proliferation assay. In vitro cell proliferation was determined using the MTT cell proliferation and viability assay kit, according to the manufacturer's instructions. Cells were cultured at a density of $5 \times 10^{3}$ cells per well in 96-well tissue culture plates and were incubated at $37^{\circ} \mathrm{C}$ for $24 \mathrm{~h}$. At the end of the culture period, the cells were washed with phosphate-buffered saline, MTT reagents were added according to the manufacturer's instructions and the absorbance was measured at $570 \mathrm{~nm}$ using an enzyme-linked immunosorbent assay plate reader (Hongda Biotech, Shanghai, China). Each experiment was repeated three times.

Establishment of orthotopic xenograft oligodendroglioma mouse models. In vivo nude mice xenografts were obtained by grafting 1,000,000 Hs683 cells to the left temporal lobes of the nude mice, as described previously (12). For experiments without BQ123 or BQ788 treatment, the mice were divided into four experimental groups: i) the vector control (VC) group $(n=9)$, where the mice were grafted with cells stably transfected with the empty pcDNA3.1 vector; ii) the ETBR group ( $n=9)$, where the mice were grafted with cells stably transfected with the pcDNA-ETBR expression vector; iii) the scramble control (SC) group ( $\mathrm{n}=9$ ), where the mice were grafted with cells stably transduced with SC shRNA; and iv) the ETBR-shRNA group $(n=18)$, where the mice were grafted with cells stably transduced with ETBR shRNA. For experiments with BQ123 or BQ788 treatment, the mice were grafted with Hs683 cells into the left temporal lobes on day zero, and subsequently injected intratumorally with saline, BQ123 or BQ788 (50 $\mu \mathrm{g} /$ mouse/day in $0.1 \mathrm{ml}$ saline) for five consecutive days from days five to nine. The mice were divided into three experimental groups: i) The normal control (NC) group ( $n=9)$, where the mice were grafted with normal Hs683 cells and subsequently received an intratumoral injection of saline; ii) the $\mathrm{NC}+\mathrm{BQ} 123$ group $(\mathrm{n}=9)$, where the mice were grafted with normal Hs 683 cells and subsequently treated with an intratumoral injection of BQ123; and iii) The $\mathrm{NC}+\mathrm{BQ788}$ group $(\mathrm{n}=18)$, where the mice were grafted with normal Hs683 cells and subsequently treated with an intratumoral injection of BQ788. The mice were monitored three times per week for signs of distress until they died or were sacrificed by cervical dislocation when the mice showed dyspnea, abnormal posture, $>20 \%$ body weight loss, difficulty with ambulation or any other clinical sign of progressive disease resulting in significant pain or distress according to the institutional guidelines of Xiangya Hospital (Central South University, Changsha, China). Twenty-seven days following grafting, all mice in the VC, SC, ETBR, NC and NC+BQ123 groups ( $\mathrm{n}=9$ each group) had died or been sacrificed, and half of the mice in the ETBR-shRNA and the NC+BQ788 groups ( $\mathrm{n}=9$ each group) were sacrificed for the purpose of comparing immunohistochemical Ki67 staining with that of the other groups. The other half of the ETBR-shRNA and the NC+BQ788 groups $(\mathrm{n}=9$ each group) were monitored until they had died or been sacrificed. All animal care, breeding, and surgical procedures were approved by the Laboratory Animal Users Committee at Xiangya Hospital, Central South University, Changsha, China.

Western blot analysis. Cells were lysed in $250 \mu \mathrm{l} 2 \mathrm{X}$ sodium dodecyl sulfate (SDS) loading buffer (62.5 mm Tris-HCl, pH 6.8; $2 \%$ SDS, $25 \%$ glycerol, $0.01 \%$ bromphenol blue, $5 \%$ 2-mercaptoethanol) and incubated at $95^{\circ} \mathrm{C}$ for $10 \mathrm{~min}$. Equal quantities of proteins $(100 \mu \mathrm{g})$ for each sample were separated by $8-15 \%$ SDS-polyacrylamide gel and blotted onto a polyvinylidene difluoride microporous membrane (Millipore, Billerica, MA, USA). Membranes were incubated for $1 \mathrm{~h}$ with a 1:500 dilution of primary antibody (anti-ETBR; N-21; Sc-21199; Santa Cruz Biotechnology) and subsequently washed and revealed using secondary antibodies with horseradish peroxidase conjugate (1:5,000, $1 \mathrm{~h}$; anti-goat IgG horseradish peroxidase conjugate; Millipore). The peroxidase was revealed using a GE Healthcare enhanced chemiluminescence kit (Beijing China). Proteins were quantified prior to being loaded onto the gel.

Immunohistochemistry. Paraffin-embedded tumor tissues were examined for Ki67 staining. Briefly, sections $(5 \mu \mathrm{m})$ 
of paraffin-embedded specimens were de-paraffinized in xylene, hydrated in a degraded series of ethanol and heated in $0.01 \mathrm{M}$ citrate buffer for $10 \mathrm{~min}$ in a microwave oven (Haier, Beijing, China). After cooling for $20 \mathrm{~min}$ and washing in PBS, endogenous peroxidase was blocked with methanol containing $0.3 \% \mathrm{H}_{2} \mathrm{O}_{2}$ for $30 \mathrm{~min}$, followed by incubation with PBS for $30 \mathrm{~min}$. Subsequently, the sections were incubated with the anti-Ki67 antibody at a dilution of 1:200, and stained using the avidin-biotin complex method. Coloration was developed by DAB containing $\mathrm{H}_{2} \mathrm{O}_{2}$, and the sections were counter-stained with hematoxylin. The number of positive cells was counted in ten high-power view fields and the percentage was calculated as follows: Ki67 positive cells/total tumor cells x 100. The slides were independently examined by two pathologists blinded to the experimental group assignment information. Cohen's $\kappa$ coefficient was calculated in order to demonstrate interobserver variability. Cohen's $\kappa$ coefficient was 0.90 in this study.

Statistical analysis. Statistical analyses were performed using SPSS for Windows 10.0 (IBM, Chicago, IL, USA). Data values were expressed as the means \pm SD. Comparisons of means among multiple groups were performed using one-way analysis of variance followed by post hoc pairwise comparisons using Tukey's tests. A log-rank test was used to compare the survival times of the mice, defined as the time to death or sacrifice. The median and mean survival times within each experimental group were estimated using the Kaplan-Meier product-limit method. A two-tailed $\mathrm{P}<0.05$ was considered to indicate a statistically significant difference.

\section{Results}

Western blot analysis. The Hs683 human glioma cell line, whose oligodendroglial origin has been extensively characterized (13), was used in this study as a cell model of oligodendroglioma. Western blot analyses revealed that ETBR was expressed in Hs683 cells (Fig. 1A). Cells were stably transfected with an ETBR expression vector in order to overexpress ETBR and the cells stably transduced with ETBR-shRNA in order to knockdown endogenous ETBR. Compared with the controls, the ETBR level in the Hs683 cells was overexpressed 2.5 fold (Fig. 1B) and knocked down by $>80 \%$ (Fig. 1C). Endogenous expression of ETs, ECE-1 and ETAR was also detected in Hs683 cells, the levels of which were unaltered by the overexpression or knockdown of ETBR in the cells (data not shown).

MTT assays. It has been reported that in oligodendroglioma cells, ETBR is functionally coupled to the activation of ERK, which is involved in cell proliferation (1). In order to investigate the role of ETBR in oligodendroglioma cell proliferation and the underlying mechanisms, Hs683 cell proliferation was examined in vitro using MTT assays. As shown in Fig. 2A, overexpression of ETBR increased cell proliferation $>2$-fold compared with the controls, which was eliminated by selective ETBR inhibitor BQ788 and ERK-specific inhibitor U0126, but not selective ETAR inhibitor BQ123. By contrast, the knockdown of endogenous ETBR decreased cell proliferation to $>0.6$-fold of the control level (Fig. 2B). A similar data trend was observed with the activation (phosphorylation) status of ERK1/2 in the cells (Fig. 3). The results indicate that

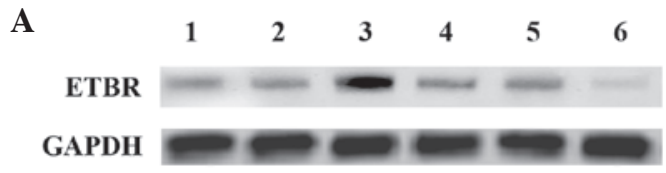

B
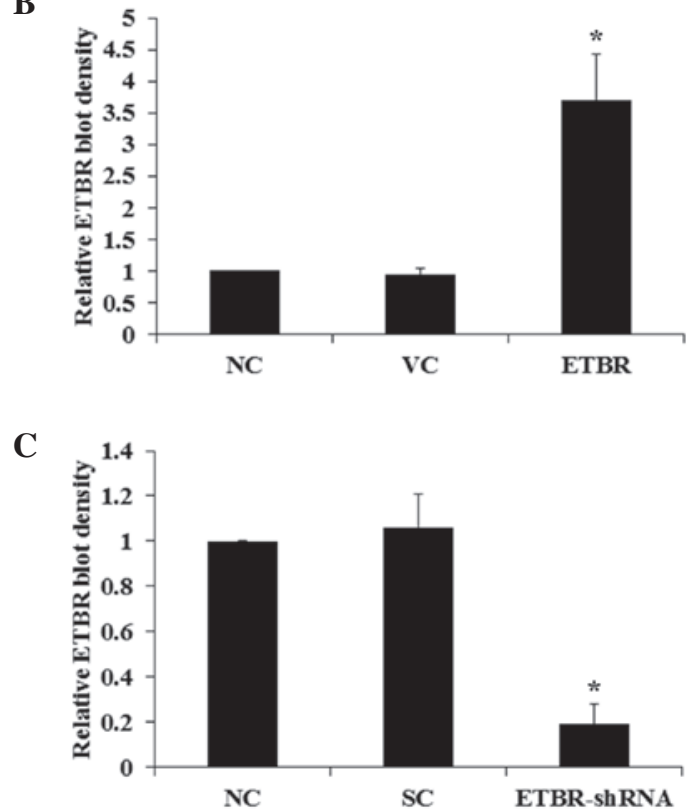

Figure 1. Endothelin B receptor (ETBR) expression in Hs683 cells (A) Lysates from Hs683 cells were subjected to western blot analyses for ETBR expression: lane 1, normal Hs683 cells (NC); lane 2, Hs683 cells stably transfected with empty pcDNA3.1 expression vector (VC); lane 3, Hs683 cells stably transfected with pcDNA-ETBR expression vector (ETBR); lane 4, normal Hs683 cells (NC); lane 5, Hs683 cells stably transduced with scramble control shRNA (SC); lane 6, Hs683 cells stably transduced with ETBR-shRNA (ETBR-shRNA). Glyceraldehyde-3-phosphate dehydrogenase (GAPDH) blotting was used as a loading control. (B and C) Protein blots were measured using densitometry. The density of the ETBR blot was normalized against that of GAPDH in order to obtain the relative blot density, which was expressed as fold changes to that of NC cells (designated as 1). ${ }^{*} \mathrm{P}<0.05$ compared with NC cells.

ETBR mediates oligodendroglioma cell proliferation by an ERK-dependent mechanism.

Orthotopic xenograph mouse model. In order to assess the role of ETBR in oligodendroglioma proliferation in vivo, an orthotopic xenograft oligodendroglioma mouse model was established. Hs683 cells stably transfected with the empty expression vector (the VC group) or the ETBR expression vector (the ETBR group), and Hs683 cells stably transduced with the SC shRNA (the SC group) or the ETBR-shRNA (the ETBR-shRNA group) were grafted orthotopically into the brains of nude mice. As shown in Fig. 4A, all mice in the ETBR group $(n=9)$ had died or been sacrificed due to the fact that they were showing signs of significant distress by day 17 following grafting. All mice in the VC and the SC groups ( $\mathrm{n}=9$ each group) had died or been sacrificed for showing signs of significant distress by day 27. By contrast, no mice in the ETBR-shRNA group $(n=9)$ died or displayed any signs of distress by day 27, and the last mouse in this group died on day 112. Log-rank tests showed that the ETBR group had a significantly shorter survival time than the controls, while the ETBR-shRNA group had a significantly longer survival time 
A

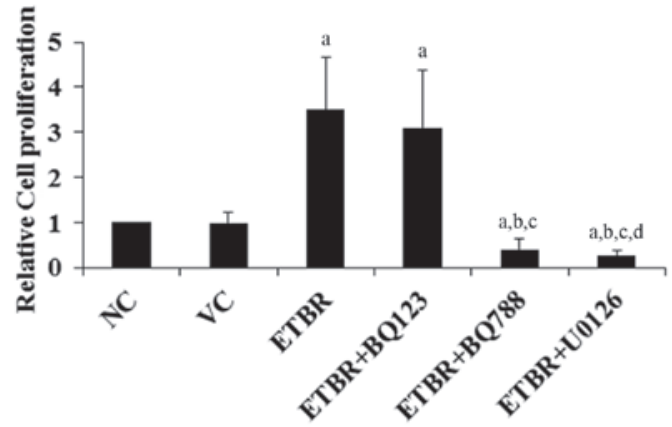

B

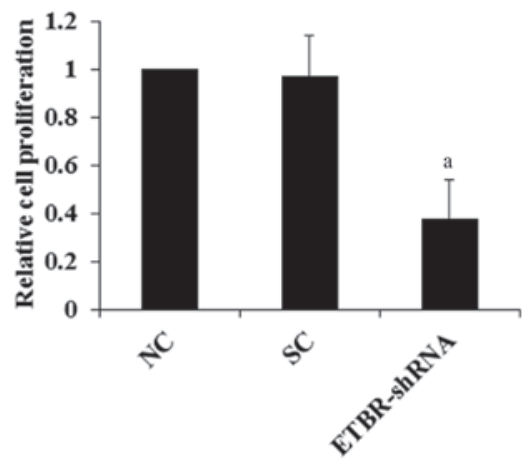

Figure 2. In vitro cell proliferation in Hs683 cells. (A) In vitro cell proliferation in normal Hs683 cells (NC), Hs683 cells stably transfected with empty pcDNA3.1 expression vector (VC), cells stably transfected with pcDNA-endothelin $B$ receptor expression vector (ETBR) and cells stably transfected with pcDNA-ETBR expression vector treated with BQ123 $(1 \mu \mathrm{M})(\mathrm{ETBR}+\mathrm{BQ123})$, BQ788 $(1 \mu \mathrm{M})(\mathrm{ETBR}+\mathrm{BQ} 788)$ or U0126 $(10 \mu \mathrm{M})(\mathrm{ETBR}+\mathrm{U} 0126)$ was determined with methlythiazoletetrazolium (MTT) assays. (B) In vitro cell proliferation in NCs, cells stably transduced with scramble control shRNA (SC), and cells stably transduced with ETBR-shRNA (ETBR-shRNA) were determined with MTT assays. Cell proliferation was expressed as fold changes to that of $\mathrm{NC}$ (designated as 1 ). ${ }^{\mathrm{a}} \mathrm{P}<0.05$ compared with $\mathrm{NC}$; ${ }^{\mathrm{b}} \mathrm{P}<0.05$ compared with ETBR; ${ }^{\mathrm{C}} \mathrm{P}<0.05$ compared with ETBR+BQ123; ${ }^{\mathrm{d}} \mathrm{P}<0.05$ compared with ETBR+BQ788.

compared with the controls. Immunohistochemical staining for Ki67, which has been widely used to assess tumor cell proliferative potential (14), demonstrated that the ETBR group had significantly increased levels of Ki67 staining in the oligodendroglioma xenograft compared with the controls, while the ETBR-shRNA group had significantly reduced Ki67 staining compared with the controls (Fig. 5).

Intratumoral injection of saline, BQ123 or BQ788. In order to investigate the therapeutic effects of the ET receptor antagonist on oligodendroglioma in vivo, normal Hs683 cells were grafted orthotopically into the brains of nude mice followed by the intratumoral injection of saline, BQ123 or BQ788. As shown in Fig. 4B, treatment with BQ788, but not BQ123, increased the survival time of the mice compared with the controls. Immunohistochemical Ki67 staining revealed that BQ788 significantly reduced Ki67 staining in the oligodendroglioma xenograft (Fig. 6).

\section{Discussion}

In the present study, it was demonstrated that ETBR mediates oligodendroglioma cell proliferation according to an ERK-dependent mechanism in vitro, and that ETBR is

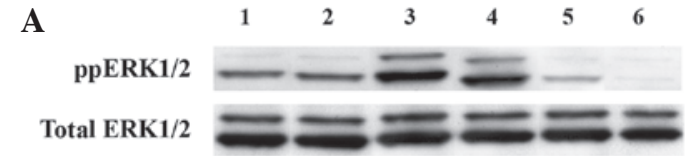

B

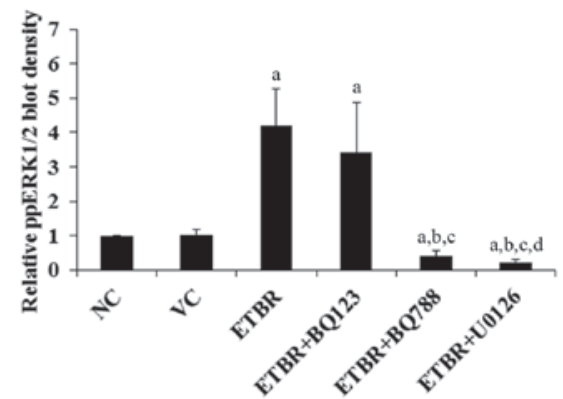

C

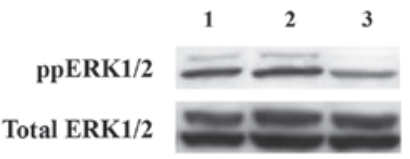

D

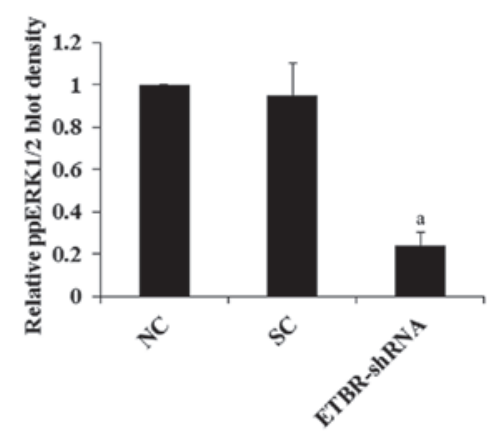

Figure 3. Western blot analysis of phosphorylated ERK (ppERK1/2) level in Hs683 cells. (A) Lysates from Hs683 cells were subject to western blot analyses in order to determine the levels of ppERK1/2 and total ERK1/2: lane 1, normal Hs683 cells (NC); lane 2, Hs683 cells stably transfected with empty pcDNA3.1 expression vector (VC); lane 3, Hs683 cells stably transfected with pcDNA-endothelin B receptor expression vector (ETBR); lane 4 , ETBR+BQ123 $(1 \mu \mathrm{M})$; lane 5, ETBR+BQ788 $(1 \mu \mathrm{M})$; lane 6, ETBR+U0126 $(10 \mu \mathrm{M})$. (B) ppERK1/2 and total ERK1/2 blots were measured using densitometry. The density of the ppERK1/2 blot was normalized against that of total ERK1/2 in order to obtain a relative ppERK1/2 blot density, which was expressed as a fold change to that of NC (designated as 1). (C) Lane 1, NC; lane 2, cells stably transduced with scramble control shRNA (SC); lane 3 , cells stably transduced with ETBR-shRNA (ETBR-shRNA). (D) Relative ppERK1/2 blot density was expressed as a fold change to that of NC (designated as 1$)$. ${ }^{\text {a }}<<0.05$ compared with $\mathrm{NC}$; ${ }^{\text {b }} \mathrm{P}<0.05$ compared with $\mathrm{ETBR}$; ${ }^{\mathrm{c}} \mathrm{P}<0.05$ compared with ETBR+BQ123 and ${ }^{\mathrm{d}} \mathrm{P}<0.05$ compared with ETBR+BQ788.

critical for oligodendroglioma proliferation and survival in vivo.

The Hs683 human glioma cell line has frequently been used as an oligodendroglioma cell model for its extensively characterized oligodendroglial origin (13). Furthermore, an orthotopic xenograft oligodendroglioma mouse model using Hs683 cells has been well established $(6,13)$. Thus, Hs683 cells were employed in the present study. A moderate expression of ETBR was detected in Hs683 cells, which allowed the effective overexpression and knockdown of ETBR to be achieved in the cells in the context of the study goals. ETs secreted from tumor cells reportedly exert autocrine/paracrine effects through the ET receptors (15). In this study, the expression levels of endogenous ETs, ECE-1 and 

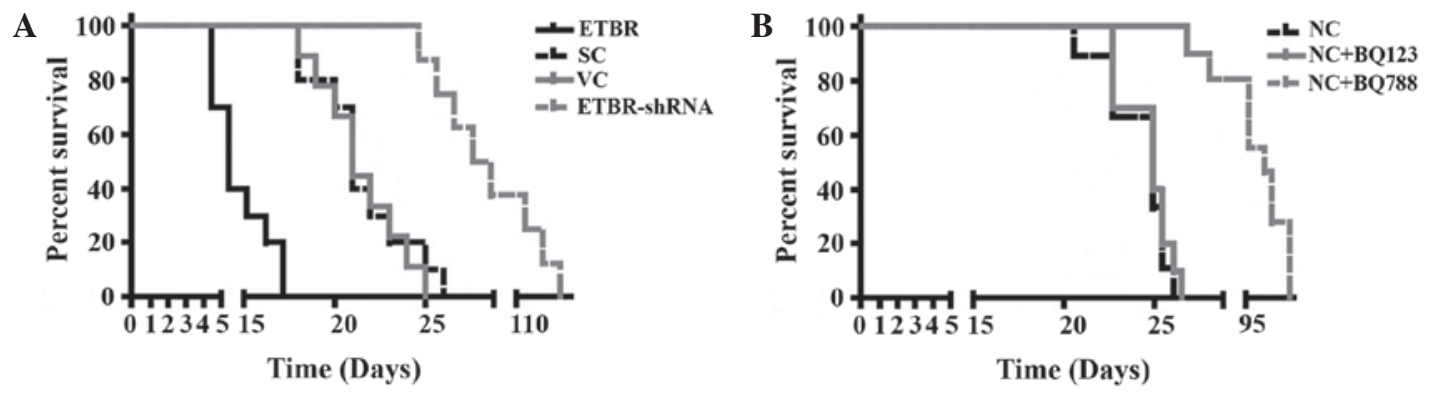

Figure 4. Kaplan-Meier curves for survival times in the orthotopic xenograft oligodendroglioma mouse model. (A) Survival curves were plotted using the Kaplan-Meier method for nude mice grafted orthotopically with $\mathrm{Hs} 683$ cells, which had been stably transfected with the empty expression vector (VC) or the ETBR expression vector (ETBR) and Hs683 cells stably transduced with the scramble control shRNA (SC) or the ETBR-shRNA (ETBR-shRNA). (B) Normal Hs683 cells (NC) were grafted orthotopically into the brains of nude mice followed by intratumoral injection of saline, BQ123 (NC+BQ123) or BQ788 (NC+BQ788). Log-rank tests were performed to compare the survival curves.

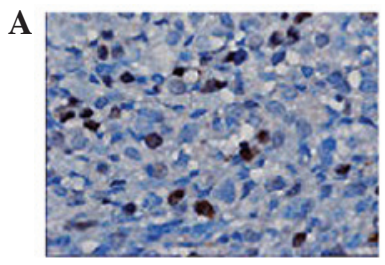

VC

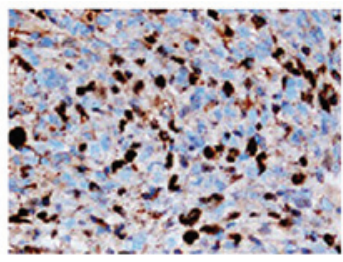

ETBR

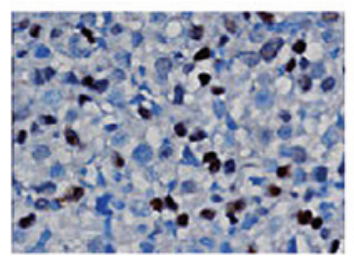

SC

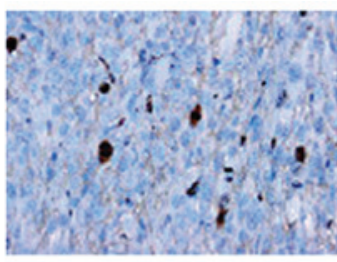

ETBR-shRNA

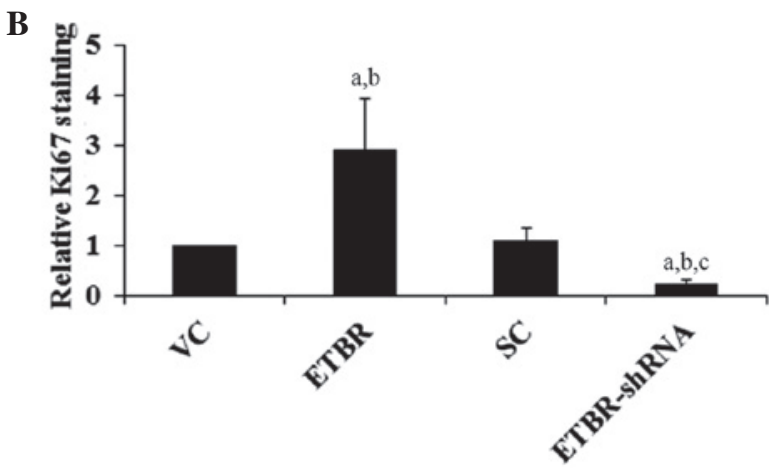

Figure 5. Immunohistochemical Ki67 staining in oligodendroglioma xenograft. (A) Hs683 cells stably transfected with the empty expression vector (VC) or the endothelin B receptor expression vector (ETBR) and Hs683 cells stably transduced with the scramble control shRNA (SC) or ETBR-shRNA were grafted orthotopically into the brains of nude mice. Immunohistochemical analyses were performed to determine Ki67 expression in the oligodendroglioma xenograft tissue on day 27 following grafting. Positive staining for Ki67 was indicated by a brownish color. Magnification, x200. (B) Ki67 positive cells were counted and the percentage was calculated as follows: Ki67 positive cells / total tumor cells x 100. Ki67 staining was expressed as fold changes to that of VC (designated as 1). ${ }^{a} \mathrm{P}<0.05$ compared with $\mathrm{VC} ;{ }^{\mathrm{b}} \mathrm{P}<0.05$ compared with $\mathrm{SC}$ and ${ }^{\mathrm{c}} \mathrm{P}<0.05$ compared with ETBR.

A

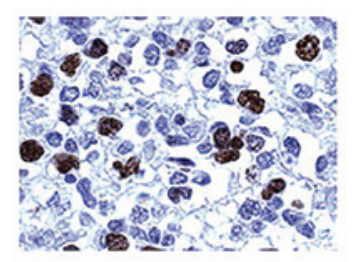

NC

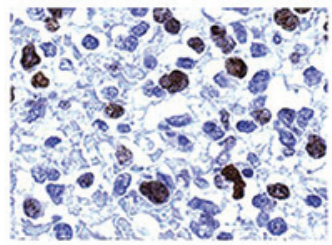

$\mathrm{NC}+\mathrm{BQ123}$

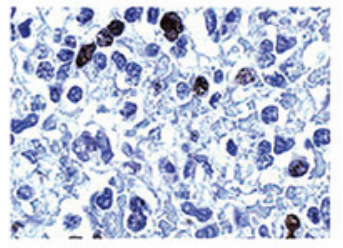

$\mathrm{NC}+\mathrm{BQ788}$
B

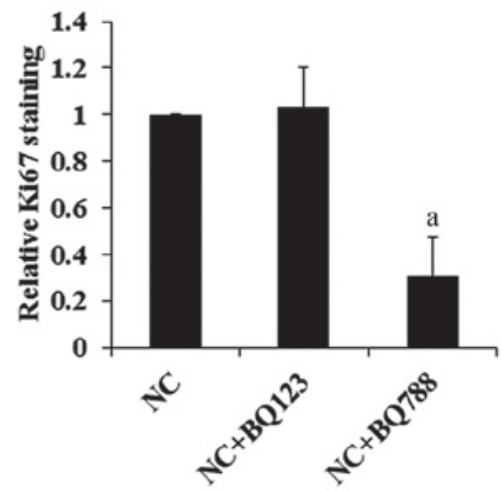

Figure 6. Immunohistochemical Ki67 staining in oligodendroglioma xenograft treated with BQ123 or BQ788. (A) Normal Hs683 cells (NC) were grafted orthotopically into the brains of nude mice followed by the intratumoral injection of saline, BQ123 (NC+BQ123) or BQ788 (NC+BQ788). Immunohistochemical analyses were performed to determine Ki67 expression in the oligodendroglioma xenograft tissue on day 27 following grafting. Positive staining for Ki67 was indicated by a brownish color. Magnification, x200. (B) Ki67 positive cells were counted and the percentage was calculated as follows: Ki67 positive cells / total tumor cells x 100. Ki67 staining was expressed as fold changes to that of NC (designated as 1 ). ${ }^{a} \mathrm{P}<0.05$ compared with NC. 
ETAR in Hs683 cells were unaltered by the overexpression or knockdown of ETBR, which enabled the investigation of the role of ETBR in the relatively stable setting of the cellular ET system.

ERK, a kinase involved in cell proliferation, is an established downstream activation target of ETBR in oligodendroglioma cells (1). In agreement with previous reports, it was observed that the overexpression of ETBR significantly increased the activation (phosphorylation) of ERK and Hs683 cell proliferation, whereas the knockdown of ETBR resulted in the opposite effects. Selective ETBR, but not an ETAR, antagonist eliminated the effects of ETBR overexpression, suggesting that ETBR, but not ETAR, mediates the proliferation of oligodendroglioma cells.

Based on the in vitro results, the role of ETBR in oligodendroglioma proliferation and survival was investigated in vivo. The majority of the experimental models used currently include either murine gliomas, which have biologic characteristics markedly different from those of human gliomas, or human glioma cell lines grafted subcutaneously onto the flanks of immunodeficient mice. In these models, diffuse invasiveness into the brain parenchyma, the hallmark of a malignant human glioma phenotype, is no longer valid (6). Thus, an Hs683 cell orthotopic xenograft mouse model was employed in the present study.

The in vivo results of the present study were in line with the in vitro findings. Immunohistochemical staining for $\mathrm{Ki} 67$, which has been widely used to assess tumor cell proliferative potential (14), indicated that the overexpression and knockdown of ETBR, respectively, increased and decreased Hs683 cell proliferation in vivo. This was confirmed by the survival results. To the best of our knowledge, this study was the first to evaluate the in vivo therapeutic effects of the ETBR antagonist on oligodendroglioma proliferation and survival. Intratumoral injection of BQ788 was used to increase local drug concentrations in the tumor xenografts for an efficient initial assessment. BQ788, but not BQ123, effectively inhibited oligodendroglioma proliferation and prolonged survival, which suggests a great potential of selective ETBR antagonists as a therapeutic alternative for oligodendrogliomas. However, the therapeutic effects of BQ788 on oligodendroglioma require further confirmation in other orthotopic xenograft mouse models.

The endothelin axis consists of three 21-amino acid peptides, ET-1, ET-2 and ET-3, two distinct receptor subtypes ETAR and ETBR, and ECEs, which catalyze the generation of biologically active ET (15). ET-1 is an established promoting factor for a wide variety of cancers (15). There is a growing body of evidence implicating ET-2 in the progression of breast cancer (16). ET-2 mRNA has been reported to be overexpressed in basal cell carcinoma compared with normal skin, an effect controlled by the Hedgehog signaling pathway (17). A study has suggested that unlike ET-1 and ET-2, ET-3 may act as a natural tumor suppressor in breast cancer (18). Pharmacological studies have demonstrated that ETAR preferentially binds to ET-1, while ETBR binds to all three ETs with a similar affinity (1). In this study, the role of ETBR in oligodendroglioma was analyzed without assessing its interaction with ETs, this requires further investigation in future studies.

In conclusion, it was demonstrated in vitro that ETBR mediates oligodendroglioma cell proliferation according to an ERK-dependent mechanism. Using an orthotopic xenograft oligodendroglioma mouse model, it was demonstrated in vivo that ETBR markedly promotes the proliferation of oligodendroglioma and that a selective ETBR antagonist may effectively inhibit the proliferation of oligodendroglioma cells and prolong survival times. This study provides a novel insight into the role of ETBR in the proliferation of oligodendroglioma and survival, and provides the first in vivo evidence that ETBR-specific antagonists may be potential therapeutic alternatives for oligodendrogliomas.

\section{Acknowledgements}

This study was supported by the Hunan Provincial Natural Science Foundation (grant no. 12F5587), Hunan, China.

\section{References}

1. Anguelova E, Beuvon F, Leonard N, et al: Functional endothelin ET B receptors are selectively expressed in human oligodendrogliomas. Brain Res Mol Brain Res 137: 77-88, 2005.

2. Chen HL, Chew LJ, Packer RJ and Gallo V: Modulation of the Wnt/beta-catenin pathway in human oligodendroglioma cells by Sox 17 regulates proliferation and differentiation. Cancer Lett 35: 361-371, 2013.

3. Ohgaki H and Kleihues P: Population-based studies on incidence, survival rates, and genetic alterations in astrocytic and oligodendroglial gliomas. J Neuropathol Exp Neurol 64: 479-489, 2005.

4. Burton E and Prados M: New chemotherapy options for the treatment of malignant gliomas. Curr Opin Oncol 11: 157-161, 1999.

5. Nutt CL, Noble M, Chambers AF and Cairncross JG: Differential expression of drug resistance genes and chemosensitivity in glial cell lineages correlate with differential response of oligodendrogliomas and astrocytomas to chemotherapy. Cancer Res 60: 4812-4818, 2000.

6. Branle F, Lefranc F, Camby I, et al: Evaluation of the efficiency of chemotherapy in in vivo orthotopic models of human glioma cells with and without $1 \mathrm{p} 19 \mathrm{q}$ deletions and in C6 rat orthotopic allografts serving for the evaluation of surgery combined with chemotherapy. Cancer 95: 641-655, 2002.

7. Levin ER: Endothelins. New Engl J Med 333: 356-363, 1995.

8. Schinelli S: Pharmacology and physiopathology of the brain endothelin system: an overview. Curr Med Chem 13: 627-638, 2006.

9. Egidy G, Eberl LP, Valdenaire O, et al: The endothelin system in human glioblastoma. Lab Invest 80: 1681-1689, 2000.

10. Paolillo M, Barbieri A, Zanassi P and Schinelli S: Expression of endothelins and their receptors in glioblastoma cell lines. J Neurooncol 79: 1-7, 2006.

11. Paolillo M, Russo MA, Curti D, Lanni C and Schinelli S: Endothelin B receptor antagonists block proliferation and induce apoptosis in glioma cells. Pharmacol Res 61: 306-315, 2010.

12. Mathieu V, De Nève N, Le Mercier M, et al: Combining bevacizumab with temozolomide increases the antitumor efficacy of temozolomide in a human glioblastoma orthotopic xenograft model. Neoplasia 10: 1383-1392, 2008.

13. Le Mercier M,Fortin S, Mathieu V, et al: Galectin 1 proangiogenic and promigratory effects in the Hs683 oligodendroglioma model are partly mediated through the control of BEX 2 expression. Neoplasia 11: 485-496, 2009.

14. Schilling H, Sehu KW and Lee WR: A histologic study (including DNA quantification and Ki-67 labeling index) in uveal melanomas after brachytherapy with ruthenium plaques. Invest Ophthalmol Vis Sci 38: 2081-2092, 1997.

15. Bagnato A, Loizidou M, Pflug BR, Curwen J and Growcott J: Role of the endothelin axis and its antagonists in the treatment of cancer. Br J Pharmacol 163: 220-233, 2011.

16. Grimshaw MJ, Hagemann T, Ayhan A, Gillett CE, Binder C and Balkwill FR: A role for endothelin-2 and its receptors in breast tumor cell invasion. Cancer Res 64: 2461-2468, 2004.

17. Tanese K, Fukuma M, Ishiko A and Sakamoto M: Endothelin-2 is upregulated in basal cell carcinoma under control of Hedgehog signaling pathway. Biochem Biophys Res Commun 391: 486-491, 2010.

18. Wiesmann F, Veeck J, Galm O, et al: Frequent loss of endothelin-3 (EDN3) expression due to epigenetic inactivation in human breast cancer. Breast Cancer Res 11: R34, 2009. 